\title{
Vanishing Balò-like lesions in multiple sclerosis
}

Balò's concentric sclerosis is a rare demyelinating disorder characterised pathologically by concentric rings of alternating demyelinated and relatively myelin preserved white matter. ${ }^{1}$ Several recent reports have shown that MRI has the potential to depict in vivo the typical appearance of Balò's concentric sclerosis lesions, thus making it possible to recognise this condition antemortem..$^{2-4}$

The figure presents the MRI obtained from a 31 year old patient with a 4 year history of clinically definite relapsingremitting multiple sclerosis. ${ }^{5}$ An MR examination performed at clinical onset of the disease showed a lesion pattern consistent with a diagnosis of multiple sclerosis. ${ }^{6}$ When the patient came to our attention, MRI was again performed, at which time eight hyperintense lesions were visible on the dual echo scans. Two of these lesions (one of which is shown in the figure) had a pattern typically seen in Balò's concentric sclerosis. ${ }^{2-4}$ On the T2 weighted images (A), these lesions showed a hypointense centre, a hyperintense inner ring, a hypointense intermediate ring, and a hyperintense outer ring. The centre of the lesions and the intermediate ring enhanced with gadolinium-DTPA (B). Diffusion weighted imaging was also performed, and the mean diffusivity values for the different lesion layers were: centre $=1.234 \times 10^{-3} \mathrm{~mm}^{2} / \mathrm{s}$; inner ring $=1.295 \times 10^{-3} \mathrm{~mm}^{2} / \mathrm{s}$; intermediate ring $=1.099 \times 10^{-3} \mathrm{~mm}^{2} / \mathrm{s}$; outer ring $=1.146 \times 10^{-3}$ $\mathrm{mm}^{2} / \mathrm{s}$. On MRI obtained 4 months later, the same eight lesions were seen on the dual echo scans, but the two lesions with a Balò's concentric sclerosis-like pattern had shrunk significantly, showed a "classic" multiple sclerosis-like appearance (D and F), and did not enhance (E).

This report shows that Balò-like lesions and multiple sclerosis-like lesions may both be present simultaneously in the same patient, and that Balò-like lesions may change over time to the classic appearance of multiple sclerosis lesions. That the two lesions we describe had a Balo'-like pattern is not only confirmed by visual inspection of the conventional MR images, but also by the changes of their mean diffusivity across the different layers. Our findings suggest that multiple sclerosis and Balò's concentric sclerosis are different manifestations of the same disease

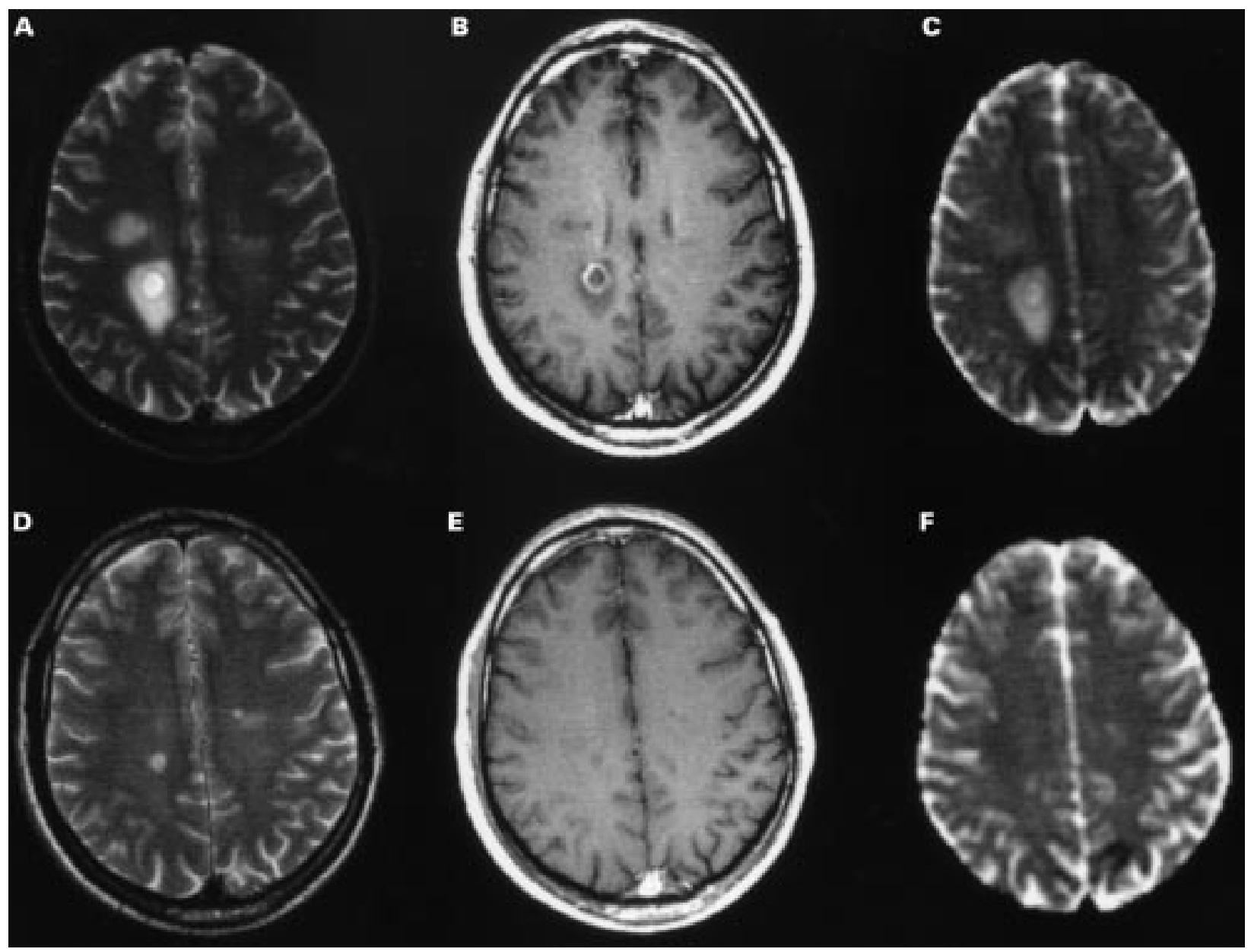

Axial T2 weighted ( $A$ and D), postcontrast $T 1$ weighted $(B$ and $E$ ) and diffusion weighted ( $C$ and $F$ ) images from a patient with multiple sclerosis. The images of the upper row show a lesion with a Balo-like pattern, and were obtained 4 months before those in the lower row where the same lesion had a more classic multiple sclerosis-like appearance. 
range rather than two separate pathological entities; this would point to a need for serial MRI before making an antemortem diagnosis of Balò's concentric sclerosis.

GIUSEPPE IANNUCCI

Neuroimaging Research Unit, Department of Neuroscience, Scientific Institute Ospedale San Raffaele, University of Milan, Via Olgettina 60, 20132 Milan, Italy

MARIO MASCALCHI

Radiodiagnostic Section, Department of Clinical Pathophysiology, University of Florence, Italy

FABRIZIO SALVI

Department of Neurological Sciences, Ospedale Bellaria, Bologna, Italy

MASSIMO FILIPPI

Neuroimaging Research Unit, Department of Neuroscience, Scientific Institute Ospedale San Raffaele, University of Milan, Via Olgettina 60, 20132 Milan, Italy
Correspondence to: Dr Massimo Filippi

1. Balò J. Encephalitis periaxialis concentrica. Archives of Neurology and Psychiatry 1928;19:242-64.

2 Kim MO, Lee SA, Choi CG, et al. Balò's concentric sclerosis: a clinical case study of brain MRI, biopsy and magnetic resonance spectroscopic findings. F Neurol Neurosurg Psychiatry 1997;62:655-8.

3 Sekijima Y, Tokuda T, Hashimoto T, et al. Serial magnetic resonance imaging (MRI) study of a patient with Balò's sclerosis treated with immunoadsorption plasmapheresis. Multiple Sclerosis 1997;2:291-4.

4 Chen CJ, Chu N, Lu C, et al. Serial magnetic resonance imaging in patients with Balò's concentric sclerosis: natural history of lesion development. Ann Neurol 1999;46:651-6.

5 Lublin FD, Reingold SC, the National Multiple Sclerosis Society (USA) Advisory Committee on Clinical Trials of New Agents in Multiple Sclerosis. Defining the clinical course of multiple sclerosis: results of an international survey. Neurology 1996;46:907-11.

6 Barkhof F, Filippi M, Miller DH, et al. Comparison of MRI criteria at first presentation to predict conversion to clinically definite multiple sclerosis. Brain 1997;120:2059-69. 